\title{
The Impact of COVID-19 on HIV Care Provided via Telemedicine-Past, Present, and Future
}

\author{
Jehan Z. Budak ${ }^{1}$ (D) John D. Scott ${ }^{1} \cdot$ Shireesha Dhanireddy $^{1} \cdot$ Brian R. Wood $^{1}$ \\ Accepted: 21 January 2021 / Published online: 22 February 2021 \\ (C) The Author(s), under exclusive licence to Springer Science+Business Media, LLC part of Springer Nature 2021
}

\begin{abstract}
Purpose of Review This review summarizes HIV care delivered via telemedicine before and during the COVID-19 pandemic and highlights areas of study to inform optimal usage of telemedicine in HIV clinical practice in the future.

Recent Findings To address barriers to care created by the COVID-19 pandemic, regulatory agencies and payors waived longstanding restrictions, which enabled rapid expansion of telemedicine across the country. Preliminary data show that providers and persons with HIV (PWH) view telemedicine favorably. Some data suggest telemedicine has facilitated retention in care, but other studies have found increasing numbers of PWH lost to follow-up and worsened virologic suppression rates despite offering video and/or telephone visits.

Summary The COVID-19 pandemic has exacerbated gaps in the HIV care continuum. To help mitigate the impact, most clinics have adopted new virtual care options and are now evaluating usage, impact, and concerns. Further research into the effects of telemedicine on HIV care and continued work towards universal access are needed.
\end{abstract}

Keywords Telemedicine $\cdot \mathrm{HIV} \cdot \mathrm{COVID}-19 \cdot \operatorname{PrEP}$

\section{Introduction}

The coronavirus disease-2019 (COVID-19) pandemic, caused by the severe acute respiratory syndrome coronavirus 2 (SARS-CoV-2), has disrupted the world and impacted every stage of the HIV care continuum. Clinics have experienced interruptions to their usual care models [1], and some have already identified decreases in viral suppression rates, especially for individuals experiencing homelessness, despite stable retention-in-care and visit volumes for in-person and telephone visits [2]. Interruptions in care and decreased viral suppression rates also have also impacted HIV transmission [3]. For many persons with HIV (PWH), the clinic serves as a medical home, and the pandemic has created substantial barriers to accessing primary care and other multidisciplinary services while trying to maintain safe physical distancing.

This article is part of the Topical Collection on eHealth and HIV

Jehan Z. Budak

jehan@uw.edu

1 Division of Allergy and Infectious Diseases, University of Washington, MS 359930, 325 9th Avenue, Seattle, WA 98104, USA
To help overcome barriers to care that have been created or exacerbated by the COVID-19 public health emergency, clinics have near universally launched or expanded telehealth options and entered a new era of virtual, distance-based medical care. Telehealth, as defined by the United States (U.S.) Health Resources and Service Administration (HRSA), is "the use of electronic information and telecommunications technologies to support and promote long-distance clinical health care, patient, and professional health-related education, public health, and health administration" [4]. Telehealth can encompass distance-based provider-to-patient interactions, providerto-provider modalities, store-and-forward communications, and mobile health applications [5]. The term telemedicine specifically refers to clinical care provided via video teleconferencing. Thus, in this review, we focus on synchronous provider-to-patient video visits, which we refer to as telemedicine.

Though telemedicine has long been technologically feasible, prior to the COVID-19 pandemic, regulatory and reimbursement barriers hindered implementation, and its use was limited to specific healthcare systems or unique situations and to research settings. When the COVID-19 pandemic reached the U.S., however, clinics across the nation needed to rapidly implement or expand telemedicine options as a way to deliver 
care while supporting stay-at-home and shelter-in-place precautions [6]. To facilitate this expansion, Congressional House Bill 6074 allowed the Department of Health and Human Services (DHHS) to temporarily waive certain telemedicine restrictions, and the Centers for Medicare \& Medicaid Services (CMS) enacted several emergency waivers to facilitate expansion of telemedicine services [7]. Examples of telemedicine restrictions waived include regulations that previously required patients to live in designated rural or medically underserved areas, that only allowed certain providers to bill for telemedicine visits, and that prohibited patients from joining video visits from home [8]. In addition, private payors amended restrictions around reimbursement, cost-sharing, prescription refill limits, and other policies. These regulatory and reimbursement changes allowed for a brisk and dramatic propagation of telemedicine services. Exemplifying the significant shift, in February 2020, 0.1\% of Medicare visits were conducted electronically, versus 43.5\% in April 2020 [9].

Although some clinics and healthcare systems were well prepared for the shift to telemedicine (i.e., with technologic platforms and protocols already in place), many were not. Most are now in the process of troubleshooting, evaluating barriers from the patient and provider perspective, devising solutions, and optimizing usage. Furthermore, the landscape once the current pandemic ends is difficult to predict; there are advantages to maintaining telemedicine options but how this will be balanced with the benefits of in-person visits and how the current regulatory waivers will change remain unclear. In this review, we (1) summarize the use of telemedicine for HIV treatment or prevention prior to the COVID-19 pandemic, (2) describe implementation strategies incorporated by clinics and health systems to sustain HIV and pre-exposure prophylaxis (PrEP) care during the COVID-19 pandemic, and (3) discuss future research questions and policy needs that will be crucial to address to inform future telemedicine usage.

\section{Telemedicine for HIV Care Before COVID-19}

Prior to the COVID-19 pandemic, PWH and their providers expressed interest in telemedicine. For example, a survey of $371 \mathrm{PWH}$ in the Houston area found that $57 \%$ of respondents were, "more likely to use telehealth for their HIV care, if available, as compared to one-on-one in-person care," and $37 \%$ answered they would use telehealth frequently or always as an alternative to clinic visits [10]. Similarly, in a survey of physicians who cared for $\mathrm{PWH}, 85 \%$ believed telemedicine could improve access and timeliness to care [11].

While PWH and HIV care providers were interested in telemedicine, before the COVID-19 public health emergency, data for the effects of telemedicine on clinical outcomes in HIV care were limited. Importantly, most models for telemedicine required a patient to travel to a clinic or other setting in order to connect to their visits, as regulations prevented them from connecting from home. Thus, comparisons to current models of telemedicine are difficult. That said, findings were generally favorable and suggested many potential benefits of telemedicine. For example, in a small study of PWH in a rural setting within a Veterans Affairs (VA) healthcare system, thirty of 32 eligible patients chose to participate in a video conferencing option [12]. Participation in telemedicine decreased travel time, led to patient satisfaction, and improved rates of flu vaccination and screening for syphilis, depression, tobacco use, and alcohol use. No statistically significant difference in retention in care or virologic suppression was observed between the groups [12]. Then, Ohl and colleagues performed a large, cluster-randomized study at clinics across three VA networks, where $1670 \mathrm{PWH}$ were offered telehealth visits [13]. Video conferencing was used uniformly, though other telehealth modalities were included and varied by site. Patients were given the option to either travel to a nearby primary care clinic to use telemedicine to connect with their HIV provider or to travel to see their HIV provider in person. Only 120 (13\%) PWH opted into the telemedicine option. The authors postulate that uptake was low because patients were already accustomed to traveling to their HIV clinic and that relatively little travel time would be saved with the telemedicine strategy (connecting from home was not an option due to the regulatory environment at the time) [13]. For those who used telemedicine, as compared to those who did not, investigators observed an increase in the number of HIV clinic visits and more frequent viral load testing, though neither the availability of telemedicine nor its usage was associated with improved viral suppression [13]. Lastly, in a retrospective chart review of PWH in rural Georgia receiving HIV care, of 385 cases reviewed (200 in-person encounters through one clinic and 185 telemedicine encounters through another), there was no statistically significant difference in mean viral load or in rates of year-round viral control between those who had inperson versus telemedicine visits [14].

Prior to the COVID-19 pandemic, some experience and data had accumulated for use of telemedicine to support HIV care in correctional settings. In a retrospective cohort study of 687 PWH in a corrections system, use of telemedicine to access HIV subspecialty care was associated with greater virologic suppression and higher CD4 T-cell counts [15]. The use of video conferencing to increase linkage to care post-release has also been studied, though, in an analysis of 144 PWH who used video conferencing with case managers in community-based organizations, telemedicine usage did not lead to a statistically significant difference in linkage rates [16]. Despite this, the authors note that telemedicine was positively received by both patients and case managers [16].

Heterogeneity of study design and telemedicine implementation logistics makes comparison between the above studies difficult. In HIV primary care settings, outcomes, including 
virologic suppression, were similar between telemedicine and in-person visits, and in some studies, telemedicine lowered barriers to care, especially barriers related to distance and travel needs. Consistent across the studies was the finding that PWH and their providers had positive attitudes toward telemedicine and were interested in its growth as an option to access care. Many expected that the numbers of individuals utilizing telemedicine in HIV and infectious diseases (ID) would continue to grow [17]. What was unanticipated, however, was the dramatic proliferation of telemedicine service implementation across the country, precipitated by the COVID-19 public health emergency.

\section{Telemedicine for HIV Ambulatory Care During the COVID-19 Pandemic}

On March 23, 2020, the DHHS published interim guidance for COVID-19 and PWH and suggested providers prescribe 90day supplies of antiretroviral medications, instead of 30-day supplies, change to mail-order medications instead of pickups, and, when possible, extend the interval and frequency with which PWH have clinic and laboratory visits [18]. In addition, the DHHS stated that video or telephone visits could replace inperson encounters for routine or non-urgent care [18].

It is clear that since the pandemic started and the regulatory and reimbursement landscape changed, telemedicine access has increased for PWH [19]. For example, Mayer et al. describe outcomes for PWH at a Boston community health center. In the first two months of 2020, their clinic had a mean of 626 in-person visits and zero telemedicine visits [20]. In March and April 2020, however, the number of in-person visits dropped to 370 and 360, respectively, while the number of telemedicine visits increased to 263 and 751 visits, respectively [20]. When telemedicine and in-person visits were combined, the mean number of visits per month by PWH was higher between March and September 2020 as compared to the same seven-month period in 2019 [20]. Despite an initial decrease in the mean number of plasma HIV RNA tests performed, monthly rates of virologic suppression at this clinic were similar between 2019 and 2020. Thus, though the number of in-person visits decreased, the authors concluded that engagement in care, based on viral suppression rates and the total number of visits, remained constant during the COVID-19 pandemic [20]. In contrast, investigators at a Midwestern academic HIV clinic serving approximately $1100 \mathrm{PWH}$, who shared operational details and quality improvement strategies from their transition to telemedicine, also assessed viral suppression and retention in care data [21]. From February to August 2020 , viral suppression rates did not change, but the number of patients lost to follow-up (which they defined as patients not seen for one year or more) increased from 34 to 59 [21].
Rogers et al. described a rapid shift to telemedicine for PWH at a newly opened LGBTQ+ clinic in Rhode Island. Anecdotally, providers had positive experiences with telemedicine and appreciated, "the opportunity to care for patients who may have more difficulty making it to in-person visits" and "the opportunity to observe patients in their homes to provide more context for their social circumstances" [22]. Patient feedback was elicited through a patient satisfaction survey, in which approximately $25 \%$ of respondents reported interest in receiving care via telemedicine, though patients also reported concerns about privacy, data breaches, billing, and insurance challenges [22]. These investigators do not report viral suppression rates, but received positive feedback from patients and providers about their experience with video visits.

In summary, most patients and providers reported positive attitudes toward telemedicine during the COVID-19 pandemic, suggesting the modality is likely to remain a part of HIV care in the future. While some studies found stable rates of virologic suppression and engagement in care [20,21], others noted increased numbers of PWH lost to follow-up [21]. The explanation for these contrasting results may be due to differences in study design, telemedicine protocols and policies, or proportion of patients with the means to access telemedicine visits. Importantly, connecting to a visit via telemedicine does not supplant the wrap-around services often needed to help an individual achieve virologic suppression [2, 23]. Thus, given that it is difficult to generalize from these studies alone and that communication with a provider is one of many elements necessary for patients to succeed with HIV treatment, more data regarding virologic suppression and retention in care during this new telemedicine era are required.

\section{Telemedicine for Pre-Exposure Prophylaxis Care During the COVID-19 Pandemic}

The pandemic accelerated the expansion of telemedicine for pre-exposure prophylaxis (PrEP) care as well. Though prior innovations in the use of telemedicine to overcome barriers to PrEP dissemination and adherence were limited to small geographic pockets or unique settings [5, 24, 25], guidance during the public health emergency encouraged telemedicine usage to deliver PrEP care [26]. Most providers have modified their PrEP practice during this time, largely through incorporation of telehealth modalities [27]. In addition, approximately 54\% noticed that their ability to test for HIV or STIs dropped [27]. This decrease in testing was mirrored by patient experiences. In an online survey of men who have sex with men in the Southern U.S. who use PrEP, during the pandemic, $47 \%$ did not receive an STI test and 35\% did not receive an HIV test [28] and a Boston clinic observed that the number of HIV tests decreased by $85.1 \%$ [29]. Data from the Boston community 
health center specializing in sexual healthcare showed that despite a rapid transition to telemedicine, COVID-19 was still associated with disruptions in PrEP care [29]. Specifically, between January and April 2020, they found that PrEP refill lapses increased by $191 \%$ and that new PrEP starts decreased by $72.1 \%$ [29]. Physical distancing and stay-at-home orders may have contributed, as a survey study found that of individuals who stopped PrEP voluntarily, 85\% stopped due to low perceived risk [27]. Though PrEP visits and prescriptions plummeted during this time, many may have stopped PrEP appropriately in the context of strict physical distancing. As the need for PrEP can fluctuate, we do not yet know whether the individuals who discontinued PrEP have re-started or reengaged in care. In addition, we need more information about the individuals who stopped PrEP due to access barriers so as to better understand how telemedicine could help relieve such barriers.

\section{Unanswered Questions and Future Directions of Telemedicine for HIV and PrEP Care}

Remote visits have several advantages beyond promoting physical distancing, including convenience; decreased travel time, expenses, or time away from work; helping individuals who fear stigma when attending clinic visits; and, importantly, helping individuals stay safe and healthy, particularly if they have risk factors for serious COVID-19 [8, 30]. Despite this, several concerns about the use of telemedicine exist, including quality of care, privacy in the home or other living situation, reimbursement, cost, medicolegal risks, increased administrative burden, and lack of institutional support [31-33]. Also, both PWH and their providers worry about decreased personal connections in their relationship [11].

One of the most pressing and significant concerns about telemedicine regards the socioeconomic disparities that prevent some individuals from accessing, and thus benefitting equally, from telemedicine. This "digital divide" is defined as the unequal access or ability to engage in care using technological means [8]. For example, female, older, lower income, and non-English-speaking patients are more likely to complete a telephone visit instead of a video visit, and non-English proficiency has been associated with a more than $50 \%$ decrease in the use of either video or telephone visits $[8,34]$. Some individuals have difficulty with video visits, often due to absence of broadband connectivity, inexperience with the technical hardware or software required, or lack of a private space in which to join a visit. Many such individuals thus rely on telephone visits to stay engaged with care; as such, telephone visits have become a crucial safety net for many patients to stay connected. The digital divide existed prior to the pandemic, and some efforts to mitigate it have been previously described, such as through individualized coaches for PWH with low health literacy to help increase their capacity to utilize the electronic health record [35]. Despite such efforts, the digital divide persists and has become more apparent due to the pandemic. A Policy Paper from the Infectious Diseases Society of America (IDSA) and HIV Medical Association (HIVMA) outlines four requirements for successful video visits (technology, technical literacy, broadband connectivity, and personal privacy), lists potential interventions to mitigate the impact of social determinants of health on telemedicine access, and calls for further research to better understand who is and who is not accessing and benefitting from telemedicine in ID and HIV clinical practice [8].

With the arrival of multiple COVID-19 vaccines, we need to start planning for the use of telemedicine after the COVID19 pandemic, both for persons with and without HIV. Many questions about the optimal usage and benefits of telemedicine and the risks of relying on telemedicine remain. Furthermore, since many of the telehealth regulatory waivers are temporary, what will the landscape of telehealth look like in a postpandemic environment? We believe telemedicine should and will remain an option after the COVID-19 pandemic and argue that regulations to support telemedicine should be made permanent. The ability to offer visits via distance and to individualize decisions about whether a person should be seen inperson or by video or telephone augments the ability to help every individual access the care they need. Payment parity for telemedicine visits is crucial and should be maintained, and payment parity for telephone visits is also necessary because those individuals who cannot access distance-based care by video often rely on phone visits to stay connected to care. Additional questions for future study that may facilitate optimal integration of telemedicine into medical care, in general and for care specifically for PWH or persons at risk for HIV, are suggested in Table 1.

\section{Conclusions}

The COVID-19 pandemic has revolutionized the practice of ambulatory medicine. We have entered an era in which we need to build telemedicine models that empower PWH and improve access to care $[30,36]$. In fact, the pandemic has accelerated differentiated care delivery in the U.S. [37, 38]. Differentiated service delivery (DSD), a care model that originated in sub-Saharan Africa, is care that is tailored to the local context and to patients' clinical status [38]. Thus, telemedicine is a form of differentiated care delivery, and we should advocate for its continued presence and expansion in the care of PWH. This advocacy should include support for the recommendations regarding the use of telehealth to strengthen the Ending the HIV Epidemic Initiative [39], implementation of 
Table 1 Potential questions and areas of study to inform future usage of telemedicine in HIV clinical care

Questions Comments and examples

Patient-focused factors

What does the patient prefer?

Is the quality of care when primarily using telemedicine equivalent to traditional in-person care? How can the quality of care be ensured and optimized?

Which clinical indications for a visit should absolutely be in-person?

Which patients are able to successfully engage in care via telemedicine? How do we prevent the new telemedicine era from excluding certain individuals?

Provider-related factors

Do providers feel comfortable performing video visits?

What are the best practices for telemedicine? How do we teach this and incorporate it into medical school and training programs?

Patient-provider interactions

Are patient-provider relationships more impersonal with telemedicine?

Do video visits increase provider implicit bias?

Telemedicine logistics and clinic operations

What is the added burden on clinics as a whole? How can clinics maximize efficiency while ensuring universal access to high-quality care via video visits?

Can wrap-around services for HIV be successfully delivered through telemedicine?
- Clinics should tailor the implementation of telehealth to their specific patient population, organizational structure, and location.

- Which patients prefer video visits, telephone visits, or in-person visits?

- More data regarding outcomes, including HIV-specific outcomes, such as viral suppression rates, and primary care outcomes, such as vaccination or cancer screening rates, are needed.

- What is missed with a video visit?

- What is the best way to ensure laboratory work or STI testing is completed? If laboratory work is ordered during a video visit, how can we ensure the patient is not billed twice (i.e., with a facility fee)?

- Mgbako et al. suggest in-person visits be prioritized for individuals newly diagnosed with HIV, new to the clinic, who are non-English-speaking, with limited access to technology, or with low health or technology literacy.

- Patients with symptoms requiring a hands-on physical exam should be seen in-person.

- Is there a best balance between frequency of video versus in-person visits for routine care of an asymptomatic individual?

- Which barriers are overcome by telemedicine, such as transportation or stigma, and which barriers remain or are exacerbated?

- Who is not engaging in telemedicine, and why not?

- How can we ensure all have access to a device and broadband connection and that those with limited English proficiency, vision, hearing, or cognitive impairment are not excluded?

- What resources will empower providers to feel more confident with telemedicine visits?

- How can training regarding telemedicine best practices be incorporated into medical school and post-graduate training so that providers entering the workforce are comfortable with this modality?

- How do patients feel about the care they receive via video-visit?

- Are patients more or less likely to stay with the same provider or clinic, and does the level of trust in their providers change?

- Are implicit biases elicited by visualizing a patient's living environment?

- Are some individuals being offered or not offered video visits simply because of demographic factors, like age or language?

- How can clinic flow (i.e., templating, check-in and check-out processes, space, and resource allocation) be streamlined for telemedicine?

- How can we maximize the patient experience while minimizing administrative and operational requirements?

- How do we bring team-based care, central to Ryan White clinics, to the telemedicine platform? the points suggested for Advancing Digital Health Equity, as outlined by the IDSA and HIVMA [8, 40], and adherence to recommendations for telemedicine for the population as a whole $[41,42]$. In addition, some have proposed that telemedicine become a Ryan White HIV/AIDS Program surrogate measure of retention in care [40, 43]. Lastly, given the structure of Ryan White clinics, we need to determine how best to provide wrap-around services and team-based care through telemedicine.

Telemedicine is at a critical juncture. It has been utilized for many years and will likely continue into the future, yet the way in which it will be utilized, how much it will be incorporated into routine clinical practice, and the degree to which it will be accepted by patients and providers in a postpandemic period is yet to be determined. Hence, we have summarized what is currently known about the use of telemedicine in PWH and how its use for PWH has grown exponentially in the midst of the pandemic and have highlighted questions and concerns which need to be addressed. Telemedicine provides an exciting opportunity to tailor care to each individual patient, to avoid traditional "one-size-fitsall" ambulatory care models, and to move toward delivering more innovative and differentiated care so that we may ultimately end the HIV epidemic. 


\section{Declarations}

Human and Animal Rights and Informed Consent This article does not contain any studies with human or animal subjects performed by any of the authors.

Conflict of Interest John Scott reports personal fees from Gilead Sciences and Premera Blue Cross. None of the other authors has any conflicts to disclose. Jehan Budak, Shireesha Dhanireddy, and Brian Wood declare that they have no conflict of interest.

\section{References}

Papers of particular interest, published recently, have been highlighted as:

- Of importance

• Of major importance

1. Qiao S, Li Z, Weissman S, Li X, Olatosi B, Davis C, et al. Disparity in HIV service interruption in the outbreak of COVID-19 in South Carolina. AIDS Behav. 2020;25:49-57. https://doi.org/10.1007/ s10461-020-03013-x.

2. Spinelli MA, Hickey MD, Glidden DV, Nguyen JQ, Oskarsson JJ, Havlir D, et al. Viral suppression rates in a safety-net HIV clinic in San Francisco destabilized during COVID-19. AIDS. 2020;34: 2328-31.

3. Stanford KA, Friedman EE, Schmitt J, Spiegel T, Ridgway JP, Moore M, et al. Routine screening for HIV in an urban emergency department during the COVID-19 pandemic. AIDS Behav. 2020;24:2757-9.

4. Telehealth Programs. Official web site of the U.S. Health Resources \& Service Administration. Available from: https://www.hrsa.gov/ rural-health/telehealth. Accessed 26 Nov 2020.

5. Touger R, Wood BR. A review of telehealth innovations for HIV pre-exposure prophylaxis (PrEP). Curr HIV/AIDS Rep. 2019;16: $113-9$.

6. Rajasekaran K. Access to telemedicine-are we doing all that we can during the COVID-19 pandemic? Otolaryngol Head Neck Surg. 2020;163:104-6.

7. H.R.6074- Coronavirus Preparedness and Response Supplemental Appropriations Act, 2020 Available at: https://www.congress.gov/ bill/116th-congress/house- bill/6074. Accessed 04 Dec 2020.

8. Wood BR, Young JD, Abdel-Massih RC, McCurdy L, Vento TJ, Dhanireddy S, et al. Advancing digital health equity: a policy paper of the Infectious Diseases Society of America and the HIV Medicine Association. Clin Infect Dis. 2020. https://doi.org/10. 1093/cid/ciaa1525.

9. Bosworth A, Ruther J, Smason LW, Sheingold S, Taplin C, Tarazi $\mathrm{W}$, et al. Medicare beneficiary use of telehealth visits: early data from the start of the COVID-19 pandemic, Office of the Assistant Secretary for Planning and Evaluation, U.S. Department of Health and Human Services. Washington, DC. July 28, 2020. Available at: https://aspe.hhs.gov/pdf-report/medicare-beneficiary-use-telehealth

10. Dandachi D, Dang BN, Lucari B, Teti M, Giordano TP. Exploring the attitude of patients with HIV about using telehealth for HIV care. AIDS Patient Care STDs. 2020;34:166-72.

11. Anderson K, Francis T, Ibanez-Carrasco F, Globerman J. Physician's perceptions of telemedicine in HIV care provision: a cross-sectional web-based survey. JMIR Public Health Surveill. 2017;3:e31.
12. Ohl M, Dillon D, Moeckli J, Ono S, Waterbury N, Sissel J, et al. Mixed-methods evaluation of a telehealth collaborative care program for persons with HIV infection in a rural setting. J Gen Intern Med. 2013;28:1165-73.

13. Ohl ME, Richardson K, Rodriguez-Barradas MC, Bedimo R, Marconi V, Morano JP, et al. Impact of availability of telehealth programs on documented HIV viral suppression: a clusterrandomized program evaluation in the Veterans Health Administration. Open Forum Infect Dis. 2019;6:ofz206.

14. Lawal FJ, Srinivasa Rao ASR, Vazquez J. Effective management of HIV in rural Georgia using telemedicine. ID Week Conference Virtual. October 21-25, 2020. Abstract 1010.

15. Young JD, Patel M, Badowski M, Mackesy-Amiti ME, Vaughn P, Shicker L, et al. Improved virologic suppression with HIV subspecialty care in a large prison system using telemedicine: an observational study with historical controls. Clin Infect Dis. 2014;59:1236.

16. Brantley AD, Page KM, Zack B, Friedrich KR, Wendell D, Robinson WT, et al. Making the connection: using videoconferencing to increase linkage to care for incarcerated persons living with HIV post-release. AIDS Behav. 2019;23:32-40.

17. Coombes CE, Gregory ME. The Current and Future Use of Telemedicine in Infectious Diseases Practice. Curr Infect Dis Rep. 2019;21:41.

18. Panel on Antiretroviral Guidelines for Adults and Adolescents. Interim Guidance for COVID-19 and Persons with HIV. Department of Health and Human Services. June 19, 2020. Available from: https://clinicalinfo.hiv.gov/en/guidelines/covid19-and-persons-hiv-interim-guidance/interim-guidance-covid-19and-persons-hiv. Accessed 01 Dec 2020.

19. Ridgway JP, Schmitt J, Friedman E, Taylor M, Devlin S, McNulty $\mathrm{M}$, et al. HIV Care continuum and COVID-19 outcomes among people living with HIV during the COVID-19 pandemic, Chicago, IL. AIDS Behav. 2020;24:2770-2.

20. Mayer KH, Levine K, Grasso C, Multani A, Gonzalez A, Biello K. Rapid migration to telemedicine in a Boston community health center is associated with maintenance of effective engagement in HIV care. ID Week Conference Virtual. October 21-25, 2020. Abstract 541.

21. Fadul N, Regan N. A Quality management project of a midwestern academic HIV clinic operation during COVID-19: implementation strategy and preliminary outcomes. ID Week Conference Virtual. October 21-25, 2020. Abstract 112.

22. Rogers BG, Coats CS, Adams E, Murphy M, Stewart C, Arnold T, et al. Development of telemedicine infrastructure at an LGBTQ+ clinic to support HIV prevention and care in response to COVID19, Providence, RI. AIDS Behav. 2020;24:2743-7.

23. Christopoulos KA, Massey AD, Lopez AM, Geng EH, Johnson MO, Pilcher CD, et al. "Taking a half day at a time:" patient perspectives and the HIV engagement in care continuum. AIDS Patient Care STDs. 2013;27:223-30.

24. Stekler JD, McMahan V, Ballinger L, Viquez L, Swanson F, Stockton J, et al. HIV Pre-exposure prophylaxis prescribing through telehealth. J Acquir Immune Defic Syndr. 2018;77:e40-2.

25. Hoth AB, Shafer C, Dillon DB, Mayer R, Walton G, Ohl ME. Iowa TelePrEP: a public-health-partnered telehealth model for human immunodeficiency virus preexposure Prophylaxis Delivery in a Rural State. Sex Transm Dis. 2019;46:507-12.

26. McCray E, Mermin J. PrEP During COVID-19. Centers for Disease Control and Prevention. Available from: https://www.hiv.gov/blog/ prep-during-covid-19. Accessed 02 Dec 2020

27. Brawley S, Dinger J, Nguyen C, Anderson J. Impact of COVID-19 related shelter-in-place orders on PrEP access, usage and HIV risk behaviors in the United States. AIDS 2020: 23rd International AIDS Conference Virtual. July 6-10, 2020. Abstract 
OADLB0101. Available from: http://programme.aids2020.org/ Abstract/Abstract/10853. Accessed 05 Dec 2020.

28. Pampati S, Emrick K, Jones J. Changes in sexual behavior and access to sexual health services due to the COVID-19 pandemic among PrEP-using MSM in the South. STD Prevention Conference Virtual. September 14-24, 2020.

29. Krakower D, Solleveld P, Levine K, Mayer K. Impact of COVID19 on HIV preexposureprophylaxis care at a Boston community health center. AIDS 2020: 23rd International AIDS Conference Virtual. July 6-10, 2020. Abstract OACLB0104. Available from: http://programme.aids2020.org/Abstract/Abstract/11755. Accessed 24 Nov 2020.

30. Mgbako O, Miller EH, Santoro AF, Remien RH, Shalev N, Olender $\mathrm{S}$, et al. COVID-19, telemedicine, and patient empowerment in HIV care and research. AIDS Behav. 2020;24:1990-3.

31. Hakim A, Gaviria-Agudelo C, Edwards K, Olson D, PIDS Telehealth Working Group. Pre-Coronavirus Disease 2019 telehealth practices among pediatric infectious diseases specialists in the United States. J Pediatric Infect Dis Soc. 2020. https://doi. org/10.1093/jpids/piaa146.

32. Green SM, Lockhart E, Marhefka SL. Advantages and disadvantages for receiving Internet-based HIV/AIDS interventions at home or at community-based organizations. AIDS Care. 2015;27:13048 .

33. Dandachi D, Lee C, Morgan RO, Tavakoli-Tabasi S, Giordano TP, Rodriguez-Barradas MC. Integration of telehealth services in the healthcare system: with emphasis on the experience of patients living with HIV. J Investig Med. 2019;67:815-20.

34. Eberly LA, Khatana SAM, Nathan AS, Snider C, Julien HM, Deleener ME, et al. Telemedicine outpatient cardiovascular care during the COVID-19 pandemic: bridging or opening the digital divide? Circulation. 2020;142:510-2.

35. Kabemba F, Davila MG, Olender S. myNYP Adopt IT: a coaching intervention to improve uptake of a personal health record (PHR). Ryan White HIV/AIDS Clinical Conference. New Orleans, LA; 2019.
36. Beima-Sofie K, Ortblad KF, Swanson F, Graham SM, Stekler JD, Simoni JM. "Keep it going if you can": HIV service provision for priority populations during the COVID-19 pandemic in Seattle, WA. AIDS Behav. 2020;24:2760-3.

37. Wilkinson L, Grimsrud A. The time is now: expedited HIV differentiated service delivery during the COVID-19 pandemic. J Int AIDS Soc. 2020;23:e25503.

38. Collins LF, Colasanti JA, Nguyen ML, Moran CA, Lahiri CD, Marconi VC, et al. The COVID-19 pandemic as a catalyst for differentiated care models to end the HIV epidemic in the U.S. applying lessons from high-burden settings. AIDS. 2020. https:// doi.org/10.1097/QAD.0000000000002746.

39. HIV.gov. About ending the HIV epidemic: plan for America. Available from: https://www.hiv.gov/federal-response/ending-thehiv-epidemic/overview. Accessed 11 Dec 2020.

40. Armstrong WS, Agwu AL, Barrette E-P, Ignacio RB, Chang JJ, Colasanti JA, et al. Innovations in HIV care delivery during the COVID-19 pandemic: policies to strengthen the ending the epidemic initiative - a policy paper of the Infectious Diseases Society of America and the HIV Medicine Association. Clin Infect Dis. 2020 https://doi.org/10.1093/cid/ciaa1532.

41. Rodriguez JA, Clark CR, Bates DW. Digital health equity as a necessity in the 21st century cures act era. JAMA. 2020;323: 2381-2.

42. Nouri S, Khoong EC, Lyles CR, Karliner L. Addressing equity in telemedicine for chronic disease management during the COVID19 pandemic. NEJM Catalyst Innovations in care Delivery. May 4, 2020. https://doi.org/10.1056/CAT.20.0123. Available from: https://catalyst.nejm.org/doi/pdf/10.1056/CAT.20.0123. Accessed 04 Dec 2020

43. Dandachi D, Freytag J, Giordano TP, Dang BN. It is time to include telehealth in our measure of patient retention in HIV care. AIDS Behav. 2020;24:2463-5.

Publisher's Note Springer Nature remains neutral with regard to jurisdictional claims in published maps and institutional affiliations. 